\title{
Numerical Simulation of Mixing by Interaction of a Vortex Ring with a Density Interface
}

\author{
Tomohiro Degawa and Tomomi Uchiyama \\ Institute of Materials and Systems for Sustainability, Nagoya University, Furo-cho, Chikusa-ku, Nagoya 464-8603, Japan
}

\begin{abstract}
This study numerically explores the mixing caused by a vortex ring launched upward into a two-layer density-stratified fluid in a rectangular tank so as to search for a highly efficient mixing method. The upper and lower fluid layers are an aqueous glycerol solution and an aqueous solution of potassium dihydrogen phosphate $\left(\mathrm{KH}_{2} \mathrm{PO}_{4}\right)$, respectively. The phenomena of vortex rings with Reynolds numbers of 4000, 6000, and 8000 were simulated. The vortex-in-cell method is used to calculate the dynamics of the upper and lower fluids, and the CIP method is used to trace the concentration of the lower fluid. The simulations reproduce the mixing process successfully and elucidate the relationship between mixing efficiency and the Reynolds number of the vortex ring.
\end{abstract}

Key words: Density-stratified fluid, mixing, vortex ring, numerical simulation, vortex method.

\section{Introduction}

Energy consumption in Japan was ranked 5th worldwide in 2015 , but Japan only supplies $5 \%$ of its own energy resources. Since most energy resources are imported, Japan must develop a stable energy supply and storage systems. Recently, Liquefied Natural Gas (LNG) has become an attractive solution because of its low environmental impact. LNG is produced around the world and is imported from multiple areas, offering benefits in terms of energy security. To store LNG efficiently, many kinds of LNG from different production areas are stored in the same LNG tank. As the density of LNG is specific to the production area and purification plant, density stratification sometimes occurs in the tank. Density-stratified LNG frequently becomes unstable over time because of variations in temperature and composition. When a density of the lower LNG layer becomes lower than that of an upper layer, sudden mixing, known as rollover, occurs. Rollover generates large amounts of vaporized gases, which cause severe damage to the tank due to over-pressurization. Thus,

Corresponding author: Tomohiro Degawa, Dr. Inf. Sc., research fields: computational fluid dynamics, multiphase flow, gas-liquid two-phase flow, vortex dynamics, high-performance computing. the prevention and elimination of LNG stratification is essential for the safe operations of LNG storage tanks. Mixing LNG by a jet issuing from a nozzle on the bottom of the tank is considered to be a promising technique for preventing and eliminating stratification in LNG storage tanks. However, influences of the density difference of the LNG and parameters of the jet, such as the Reynolds number and the issuing angle, on the mixing processes have not been clarified because it is not possible to visualize the phenomena occurring in LNG storage tanks.

Mixing induced by jets of density-stratified fluids in a tank has been studied previously via laboratory-based experiments [1-4]. One of the authors [5] performed a laboratory-based experiment to investigate mixing induced by a jet issuing into a two-layer density-stratified fluid in a rectangular tank. The upper fluid was water and the lower fluid was an aqueous solution of sodium chloride $(\mathrm{NaCl})$, and the lower fluid was injected straight upward from a nozzle on the bottom of the tank. The studies confirmed that the behavior of the jet relative to the density interface is determined by the Reynolds number of the jet and the mass concentration of the lower fluid. Numerical simulations were also conducted under the same conditions, confirming the 
validity of the simulation method [6]. The current authors carried out experiments [7] and numerical simulations [8] to investigate mixing with a jet is issuing upward at an angle in a cylindrical tank. The angle between the centerline of the jet and the tank bottom was $60^{\circ}$. The studies clarified the appearances of jet-induced flows and internal density currents at the density interface. Simulations [8] successfully complemented this experimental investigation.

Vortex rings, formed by instantaneous jet eruptions, can transport mass and momentum efficiently through its convection with the self-induced velocity. This high transport ability has attracted much research attention to matter transport via vortex rings. Domon et al. [9] conducted an experimental study on the transport of spherical resin particles (mean diameter: $0.4 \mathrm{~mm}$, specific weight: 1.02). The motion of particles trapped into a vortex ring launched into quiescent water was visualized. Uchiyama et al. [10] experimentally explored the generation and transport of clusters of polyacetal particles (mean diameter: $1.52 \mathrm{~m}$, specific weight: 1.4) by a vortex ring. Bernal et al. [11] experimentally and numerically investigated the formation and propagation of a distinct droplet caused by the collision of a vortex ring with the density interface in a two-layer density-stratified fluid. They also experimentally studied the formation and propagation of a droplet by a vortex ring through three-layer density-stratified fluids [12]. The middle layer in this study was relatively thin. They found that a droplet of lower fluid forms, and is coated with the middle fluid.

LNG mixing by vortex rings is considered to be able to lead to efficiency improvements and energy savings. Interactions between the density interface and a vortex ring launched vertically downward from the upper layer have been studied experimentally and numerically. Linden [13] performed laboratory-based experiments to investigate the relationship between the Froude number and the penetration depth of the vortex ring. Olsthoorn and Dalziel [14] performed experiments on mixing of two-layer density-stratified fluids by sequentially launched vortex rings. They measured the change in the density of the mixed fluid in the upper layer and the height of the density interface. Tsunoda et al. [15] experimentally observed the time variation of three-dimensional structures of a vortex ring as it collides with the density interface. The effects of the density difference and Reynolds number of the vortex ring were measured. Dahm et al. [16] experimentally and numerically investigated the effect of the density ratio and inertia of the vortex ring on the interaction between the vortex ring and the density interface.

In these experiments [14-16], the vortex ring was launched from the upper layer and moves straight downward toward the density interface. If the existing equipment such as pumps and pipelines for LNG delivery is utilized for mixing in LNG tanks, however, vortex rings will be launched from lower layer and move upward in the tank. Song et al. [17] investigated the deformation of the water surface and the generations of free surface waves caused by collision with a vortex ring. The vortex ring was launched vertically upward from the tank bottom and collided with the water surface. They observed three different wave patterns, depending on the strength of the vortex ring. Marcus and Bell [18] simulated the interaction of a vortex ring with the density interface using axisymmetric coordinates. The deformation of the vortex ring during the interaction was simulated. However, the mixing of the lower and upper fluid was not measured in these studies [11, 12, 17, 18].

This study numerically explores the mixing of two-layer density-stratified fluid in a rectangular tank by a vortex ring. In the simulations, the vortex ring is launched from the lower layer into the two-layer density-stratified fluid which consists of an aqueous glycerol solution and an aqueous solution of potassium dihydrogen phosphate $\left(\mathrm{KH}_{2} \mathrm{PO}_{4}\right)$ as in the experimental study by Tsunoda et al. [15]. The vortex in cell method is applied to fluid dynamics simulations that shed light on the practicality of vortex 
rings for mixing density-stratified fluids.

\section{Basic Equation and Numerical Method}

\subsection{Basic Equation}

The simulation uses the Boussinesq approximation, which assumes that the difference in density appears only affects the gravitational term. In this case, the mass and momentum conservation equations are expressed as:

$$
\nabla \cdot \boldsymbol{u}=0
$$

$$
\frac{\partial \boldsymbol{u}}{\partial t}+(\boldsymbol{u} \cdot \nabla) \boldsymbol{u}=-\frac{1}{\rho} \nabla p+v_{G} \nabla^{2} \boldsymbol{u}-\frac{\rho_{G}-\rho}{\rho_{G}} \boldsymbol{g}
$$

where, $\rho_{G}$ and $v_{G}$ are the density and kinematic viscosity of an aqueous glycerol solution, respectively, and $\boldsymbol{g}$ is gravitational acceleration. The density $\rho$ in Eq. (2) is given by the following equation:

$$
\rho=(1-C) \rho_{G}+C \rho_{K}
$$

Here, $C$ and $\rho_{K}$ are the concentration and density of the aqueous $\mathrm{KH}_{2} \mathrm{PO}_{4}$ solution, respectively, and $C$ is governed by the following convection-diffusion equation:

$$
\frac{\partial C}{\partial t}+(\boldsymbol{u} \cdot \nabla) C=\kappa \nabla^{2} C
$$

where $\kappa$ is the diffusion coefficient of the aqueous $\mathrm{KH}_{2} \mathrm{PO}_{4}$ solution [19].

Taking the curl of Eq. (2) and substituting Eq. (1) into the resulting equation, the following vorticity equation is derived:

$$
\begin{aligned}
\frac{\partial \boldsymbol{\omega}}{\partial t}+\nabla \cdot(\boldsymbol{u} \boldsymbol{\omega}) & =\nabla \cdot(\boldsymbol{\omega u}) \\
& +v \nabla^{2} \boldsymbol{\omega}-\nabla \times \frac{\rho_{G}-\rho}{\rho_{G}} \boldsymbol{g}
\end{aligned}
$$

where, $\boldsymbol{\omega}$ is the vorticity.

$$
\boldsymbol{\omega}=\nabla \times \boldsymbol{u}
$$

According to the Helmholtz theorem, any vector field can be represented as the summation of the gradient for a scalar potential $\phi$ and the curl of a vector potential $\boldsymbol{\psi}$. Thus, the velocity $\boldsymbol{u}$ is written as:

$$
\boldsymbol{u}=\nabla \phi+\nabla \times \boldsymbol{\psi}
$$

The velocity calculated from Eq. (7) remains unaltered even when any gradient of scalar potential function is added to $\boldsymbol{\psi}$. To remove this arbitrariness, a solenoidal condition is imposed on $\boldsymbol{\psi}$.

$$
\nabla \cdot \boldsymbol{\psi}=0
$$

When substituting Eq. (7) into Eq. (1), the following equation is obtained:

$$
\nabla^{2} \phi=0
$$

Taking the curl of Eq. (7) and substituting Eq. (1) into the resultant equation, the vector Poisson equation is derived:

$$
\nabla^{2} \boldsymbol{\psi}=-\boldsymbol{\omega}
$$

\subsection{Vortex in Cell Method}

The vorticity field is discretized by vortex elements, and the computational domain is divided into a computational grid. The position vector and vorticity for the vortex element $v$ are assumed to be $\boldsymbol{x}_{v}=\left(x_{v}, y_{v}, z_{v}\right)$ and $\boldsymbol{\omega}_{v}$, respectively. The Lagrangian form of the vorticity equation, Eq. (5), is written as follows:

$$
\begin{gathered}
\frac{\partial \boldsymbol{x}_{v}}{\partial t}=\boldsymbol{u}\left(\boldsymbol{x}_{v}\right) \\
\frac{\partial \boldsymbol{\omega}_{v}}{\partial t}=\nabla \cdot\left(\boldsymbol{\omega}\left(\boldsymbol{x}_{v}\right) \boldsymbol{u}\left(\boldsymbol{x}_{v}\right)\right) \\
+\nu \nabla^{2} \boldsymbol{\omega}\left(\boldsymbol{x}_{v}\right)-\nabla \times \frac{\rho_{G}-\rho\left(\boldsymbol{x}_{v}\right)}{\rho_{G}} \boldsymbol{g}
\end{gathered}
$$

When the position and vorticity of a vortex element are known at time $t$, the values at $t+\Delta t$ can be computed from Eqs. (11) and (12). If $\boldsymbol{\omega}$ is defined at position $\boldsymbol{x}_{k}=\left(x_{k}, y_{k}, z_{k}\right)$, the vorticity $\boldsymbol{\omega}$ is assigned to $\boldsymbol{x}_{k}$, or a vortex element with vorticity $\boldsymbol{\omega}_{v}$ is redistributed onto $\boldsymbol{x}_{k}$.

$\boldsymbol{\omega}\left(\boldsymbol{x}_{v}\right)$

$=\sum_{v}^{N_{v}} \omega_{v} W\left(\frac{x_{k}-x_{v}}{\Delta x}\right) W\left(\frac{y_{k}-y_{v}}{\Delta y}\right) W\left(\frac{z_{k}-z_{v}}{\Delta z}\right)$

where, $N_{v}$ is the number of vortex elements, and $\Delta x, \Delta y$, and $\Delta z$ are the grid widths. For the redistribution function $W$, the following equation is employed [20]:

$$
W(\varepsilon)= \begin{cases}1-2.5 \varepsilon^{2}+1.5|\varepsilon|^{3} & |\varepsilon| \leq 1 \\ (2-|\varepsilon|)^{2}(1-|\varepsilon|) / 2 & 1<|\varepsilon| \leq 2 \\ 0 & |\varepsilon|>2\end{cases}
$$




\subsection{Constrained Interpolation Profile Method}

The convection-diffusion equation for the concentration of $\mathrm{KH}_{2} \mathrm{PO}_{4}$, Eq. (4), is solved by the Constrained Interpolation Profile (CIP) method [21] to represent the density interface with fine resolution. In the CIP method, the concentration and its gradient are simultaneously propagated to prevent numerical diffusion. When convection terms are non-linear, the CIP method divides Eq. (4) into two phases, namely the convection phase and non-convection phase, to calculate the time evolution of the concentration. The concentration gradient $\nabla C$ and diffusion term $\kappa \nabla^{2} C$ on the right-hand side of Eq. (4) are written as:

$$
\begin{gathered}
C^{\prime} \equiv \nabla C \\
D \equiv \kappa \nabla^{2} C
\end{gathered}
$$

In the convection phase, the concentration and its gradient are propagated from the following equations in a semi-Lagrangian manner.

$$
\begin{gathered}
\frac{\partial C}{\partial t}+(\boldsymbol{u} \cdot \nabla) C=0 \\
\frac{\partial \boldsymbol{C}^{\prime}}{\partial t}+(\boldsymbol{u} \cdot \nabla) \boldsymbol{C}^{\prime}=0
\end{gathered}
$$

$C$ and $C^{\prime}$ on every grid point are updated from the values upstream of the grid points like below:

$$
\begin{aligned}
C(x, y, z, t+\Delta t) & \\
& =C\left(x-u_{x} \Delta t, y-u_{y} \Delta t, z\right. \\
& \left.-u_{z} \Delta t, t\right)
\end{aligned}
$$

$C$ and $\boldsymbol{C}^{\prime}$ at the upstream-point $\left(x-u_{x} \Delta t, y-\right.$ $\left.u_{y} \Delta t, z-u_{z} \Delta t\right)$ are estimated from the interpolation polynomial $F$.

$$
\begin{aligned}
F(x, y, x) & =\sum_{i=0, j=0, k=0}^{i+j+k \leq 3} c_{i j k} x^{i} y^{j} z^{k} \\
& +c_{310} x^{3} y+c_{301} x^{3} z+c_{130} x y^{3} \\
& +c_{031} y^{3} z+c_{103} x z^{3}+c_{013} y z^{3} \\
& +c_{211} x^{2} y z+c_{121} x y^{2} z+c_{112} x y z^{2} \\
& +c_{311} x^{3} y z+c_{131} x y^{3} z+c_{113} x y z^{3}
\end{aligned}
$$

For the coefficients $c_{i j k}$, the equations given in appendix A of Ref. [22] are used. In the non-convection phase, following two equations are calculated with a forward-time, center-space scheme.

$$
\begin{gathered}
\frac{\partial C}{\partial t}=D \\
\frac{\partial \boldsymbol{C}^{\prime}}{\partial t}=\nabla D-\left(\boldsymbol{C}^{\prime} \cdot \nabla\right) \boldsymbol{u}
\end{gathered}
$$

The second-order central-finite-difference method is applied for spatial difference calculations and the first-order forward Euler method is applied for time integration.

\subsection{Computational Procedures}

The time evolution of the flow is simulated by the following procedure:

(1) Calculate the convection of the vortex elements from Eq. (11).

(2) Redistribute $\boldsymbol{\omega}_{v}$ onto grid points from Eq. (13).

(3) Calculate the time rate of change for $\boldsymbol{\omega}$ from Eq. (12).

(4) Calculate $\boldsymbol{\psi}$ from Eq. (10).

(5) Calculate $\phi$ from Eq. (9).

(6) Calculate $\boldsymbol{u}$ from Eq. (7).

(7) Recalculate the vorticity $\boldsymbol{\omega}$ by taking the curl of $\boldsymbol{u}$ to satisfy the solenoidal condition for $\boldsymbol{\omega}$ [23].

(8) Calculate convection phase for $C$ from Eq. (17).

(9) Calculate convection phase for $\boldsymbol{C}^{\prime}$ from Eq. (18).

(10) Calculate non-convection phase for $C$ from Eq. (21).

(11) Calculate non-convection phase for $C^{\prime}$ from Eq. (22).

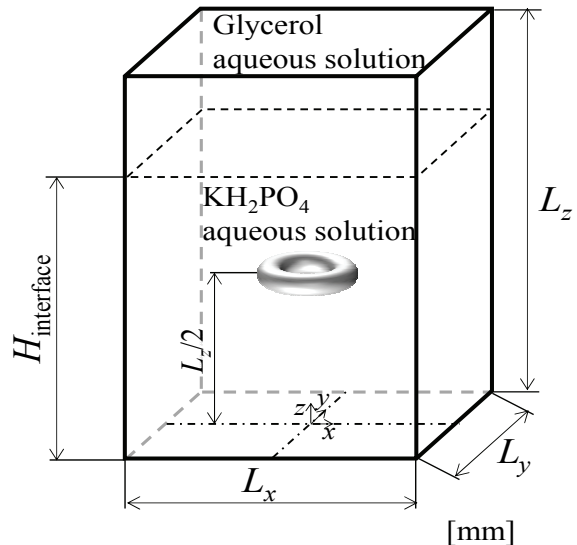

Fig. 1 Two-layer density-stratified fluid and vortex rings in a rectangular tank. 


\section{Computational Condition}

At the initial time $t=0$, a two-layer density-stratified fluid is at equilibrium in a rectangular tank, as shown in Fig. 1. The upper and lower fluids are an aqueous glycerol and aqueous $\mathrm{KH}_{2} \mathrm{PO}_{4}$ solutions, respectively. The kinetic viscosity and density of the upper fluid, $v_{G}$ and $\rho_{G}$, are $1.037 \times 10^{-6} \mathrm{~m}^{2} / \mathrm{s}$ and $1,004.9 \mathrm{~kg} / \mathrm{m}^{3}$, respectively. Those of the lower fluid, $v_{K}$ and $\rho_{K}$, are $1.010 \times$
$10^{-6} \mathrm{~m}^{2} / \mathrm{s}$ and $1,020.0 \mathrm{~kg} / \mathrm{m}^{3}$, respectively. The resulting density ratio of the lower to upper fluids $\left(=\rho_{G} / \rho_{K}\right)$ is 1.015 and the Atwood number At $\left(=\left(\rho_{K}-\rho_{G}\right) /\left(\rho_{K}+\rho_{G}\right)\right)$ is 0.0075 .

The size of the tank $L_{x} \times L_{y} \times L_{z}$ is $240 \times 240 \times$ $320 \mathrm{~mm}$. The origin of the coordinates is set at the center of the tank bottom. The density interface is positioned $3 L_{z} / 4$ above the tank bottom. A vortex ring is initially placed at $\left(x_{r}, y_{r}, z_{r}\right)=\left(0,0, L_{z} / 2\right)$ and its azimuthal vorticity $\omega_{\theta}$ is given by:

$$
\omega_{\theta}=\frac{\Gamma}{\pi a^{2}} \exp \left[-\frac{\left\{\sqrt{\left(x-x_{r}\right)^{2}+\left(y-y_{r}\right)^{2}}-R\right\}^{2}+\left(z-z_{r}\right)^{2}}{a^{2}}\right]
$$

where, $R, a$, and $\Gamma$ are the ring radius, core radius, and circulation of the vortex ring. The radius and core radius of the vortex ring are set to $13.2 \mathrm{~mm}$ and $6 \mathrm{~mm}$, respectively. The scalar potential $\phi$ is set to zero in the entire domain, and accordingly the solution of Eq. (9) is omitted. The tank is divided into $240 \times 240 \times$ 320 cubic grid cells. A staggered grid is employed to ensure consistency between the discretized equations and prevent numerical oscillation of the solution. A non-slip condition is imposed on all boundaries. Vortex rings of Reynolds number $\left(=\Gamma / \nu_{K}\right) 4000$, 6000 and 8000 were simulated for the duration from $t^{*}=t /\left(R^{2} / \Gamma\right)=0$ to 500 . The Froude number Fr is defined as $\sqrt{\Gamma^{2} /\left(8 R^{3} g\right)}$, based on the circulation $\Gamma$ and diameter of the vortex ring $2 R$. The Froude numbers in the simulations were $0.256,0.384$, and 0.512. The Richardson numbers $\mathrm{Ri}\left(=\mathrm{At} / \mathrm{Fr}^{2}\right.$ ) were $0.113,0.0505$, and 0.0284 . This study examines the effect of the circulation of the vortex ring. Because the jet velocity is the only variable for the operation of LNG storage tanks, thus the circulation of the vortex rings is the main controllable parameter in the generation of vortex rings by jet eruptions.

\section{Results and Discussion}

\subsection{Vortex Ring Behavior}

Fig. 2 shows the vorticity magnitude $|\boldsymbol{\omega}|$ and the density interface at $\mathrm{Re}=4,000$. The density interface is defined as the position at which the concentration of the aqueous $\mathrm{KH}_{2} \mathrm{PO}_{4}$ solution $C$ is 0.5 , and the interface is depicted in the figure as a gray surface. A quarter of the area of the interface $(x \geq 0, y \geq 0)$ is removed in the figure for easy inspection. At first, the vortex ring moves upward toward the density interface. As the vortex ring reaches the interface, the interface is entrained into the vortex ring. The vortex ring of lower fluid loses its kinetic energy via convection to the upper fluid and eventually sinks back to the lower layer, entraining the upper fluid into the lower layer. No vorticity appears in the upper layer, meaning that the vortex motion occurs only below the interface. Fig. 3 shows the vorticity magnitude $|\boldsymbol{\omega}|$ and the density interface at $\operatorname{Re}=6,000$. The vortex ring reaches a higher position than that at $\operatorname{Re}=4,000$ and collapses in the upper layer. In contrast to the results at $\mathrm{Re}=$ 4,000 , vortex motion does appear above the interface. With the Reynolds number further increased to $\mathrm{Re}=$ 8,000 , the vortex ring convects toward the upper wall while maintaining its shape even after penetrating the density interface, as shown in Fig. 4. The vortex ring collides with the wall, spreads out, and gradually sinks in the upper layer.

Figs. 5 and 6 show the concentration of the lower fluid $C$ in the central vertical $(x-z)$ cross-section passing through the tank center at four different time 

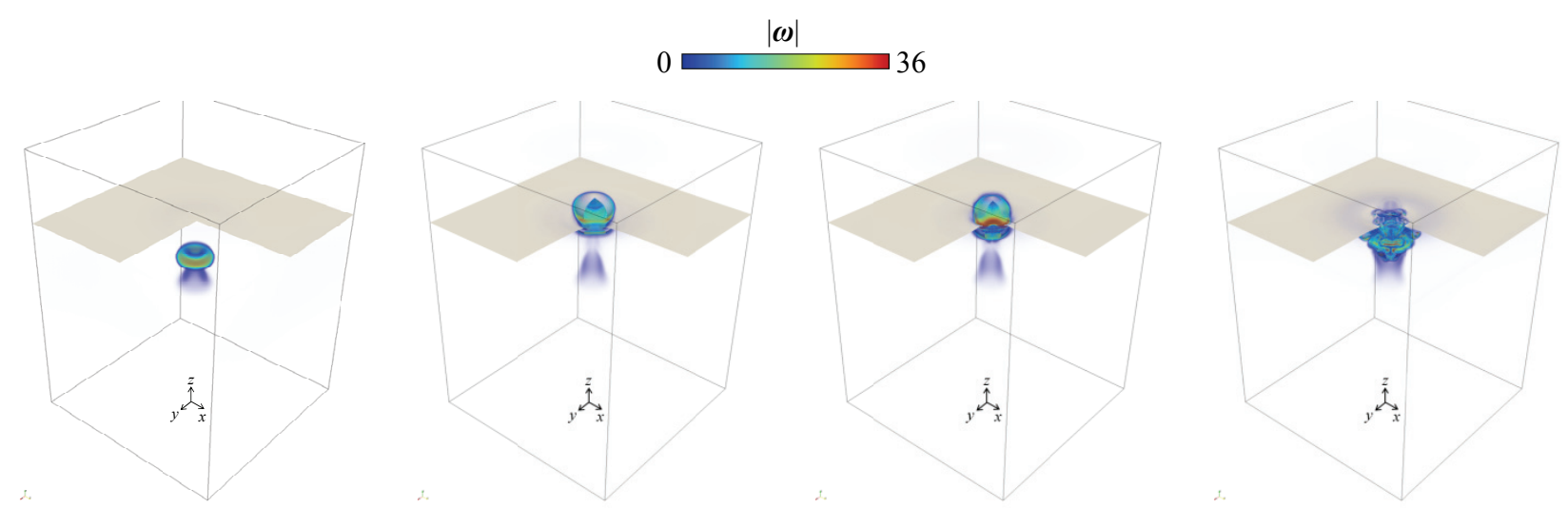

(a) $t^{*}=15.0$

(b) $t^{*}=54.3$

(c) $t^{*}=65.6$

$(\mathrm{d}) t^{*}=93.7$

Fig. 2 Time variation of vorticity magnitude and density interface at $\operatorname{Re}=4,000$.
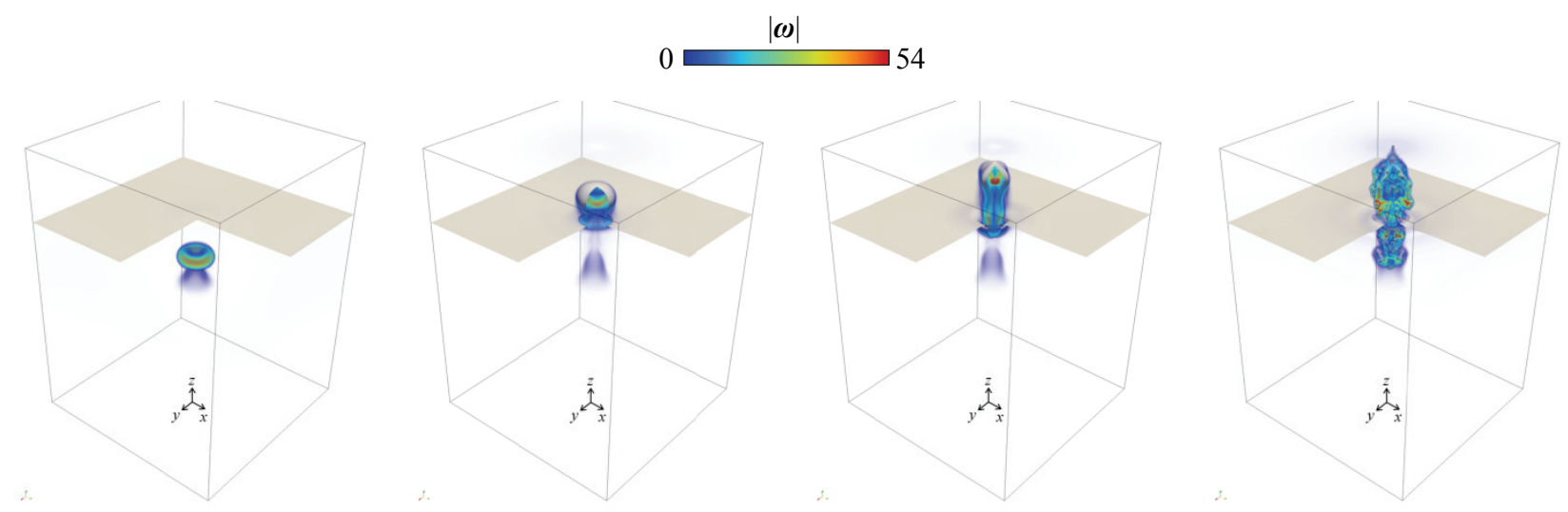

(a) $t^{*}=15.0$

(b) $t^{*}=54.3$

(c) $t^{*}=74.9$

$(\mathrm{d}) t^{*}=112$

Fig. 3 Time variation of vorticity magnitude and density interface at $\operatorname{Re}=\mathbf{6 , 0 0 0}$.

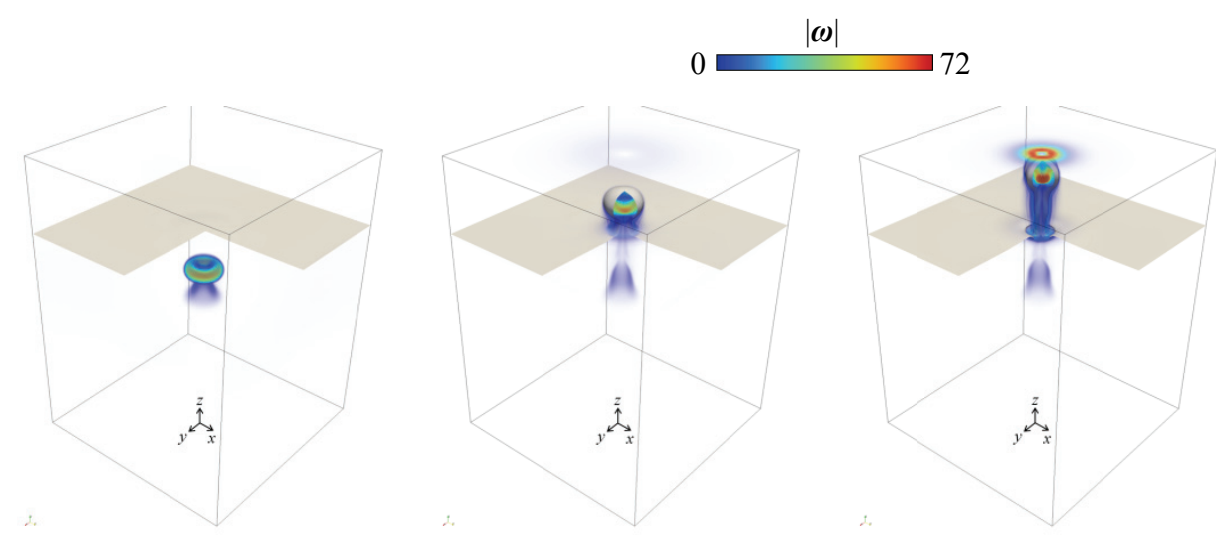

(a) $t^{*}=15.0$

(b) $t^{*}=54.3$

(c) $t^{*}=74.9$

$(\mathrm{d}) t^{*}=131$

Fig. 4 Time variation of vorticity magnitude and density interface at $\operatorname{Re}=8,000$. 


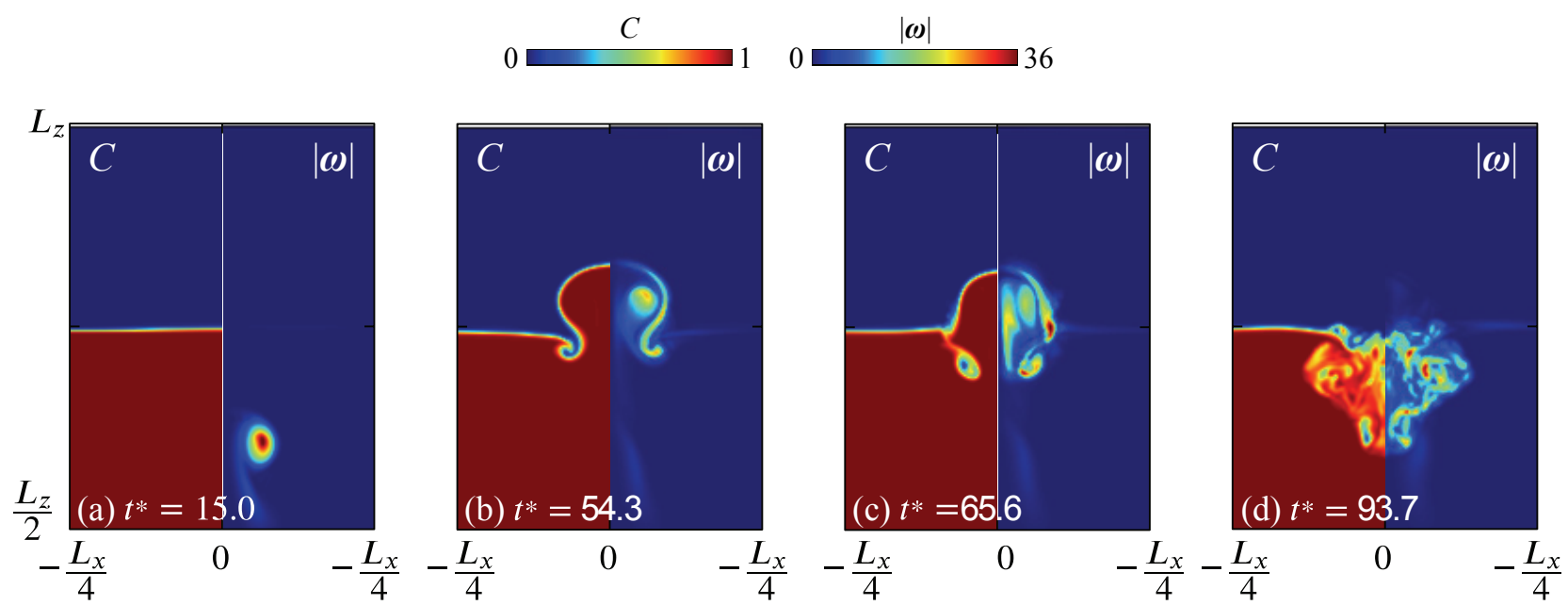

Fig. 5 Time variation of vorticity magnitude and concentration in the central vertical cross-section at $\operatorname{Re}=4,000$.

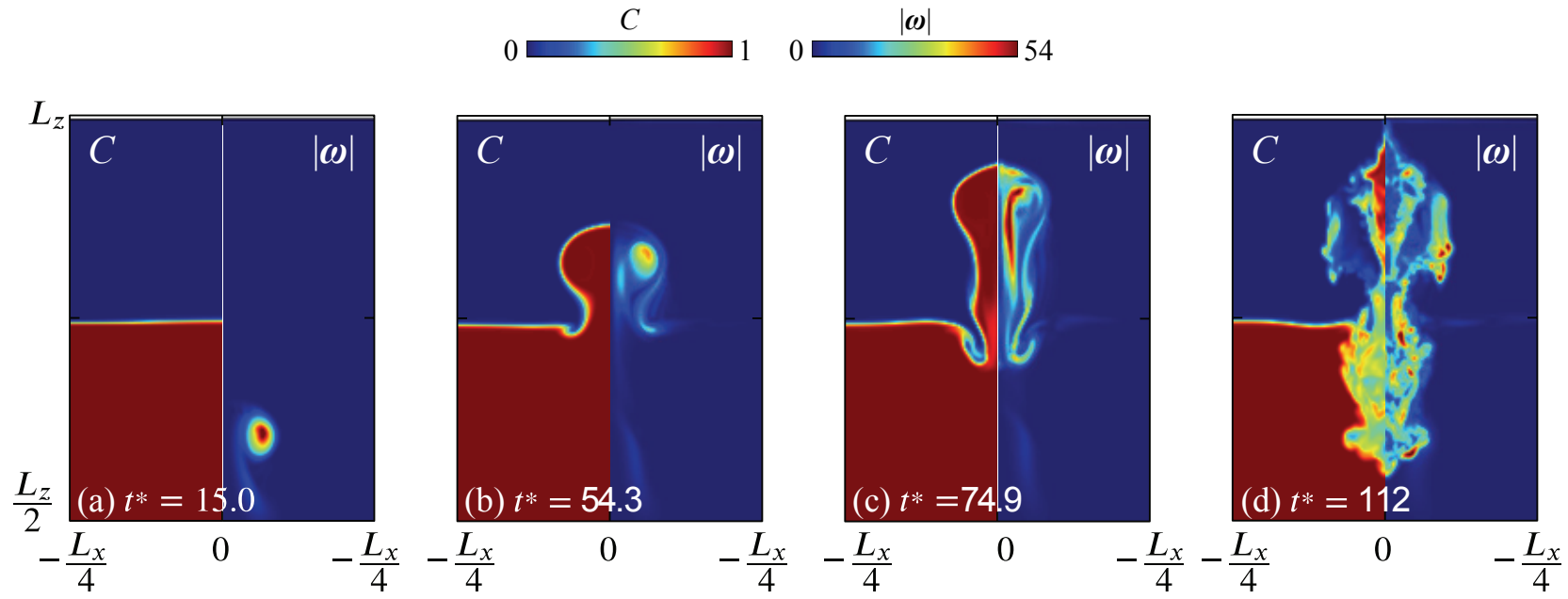

Fig. 6 Time variation of vorticity magnitude and concentration in the central vertical cross-section at $\operatorname{Re}=6,000$.

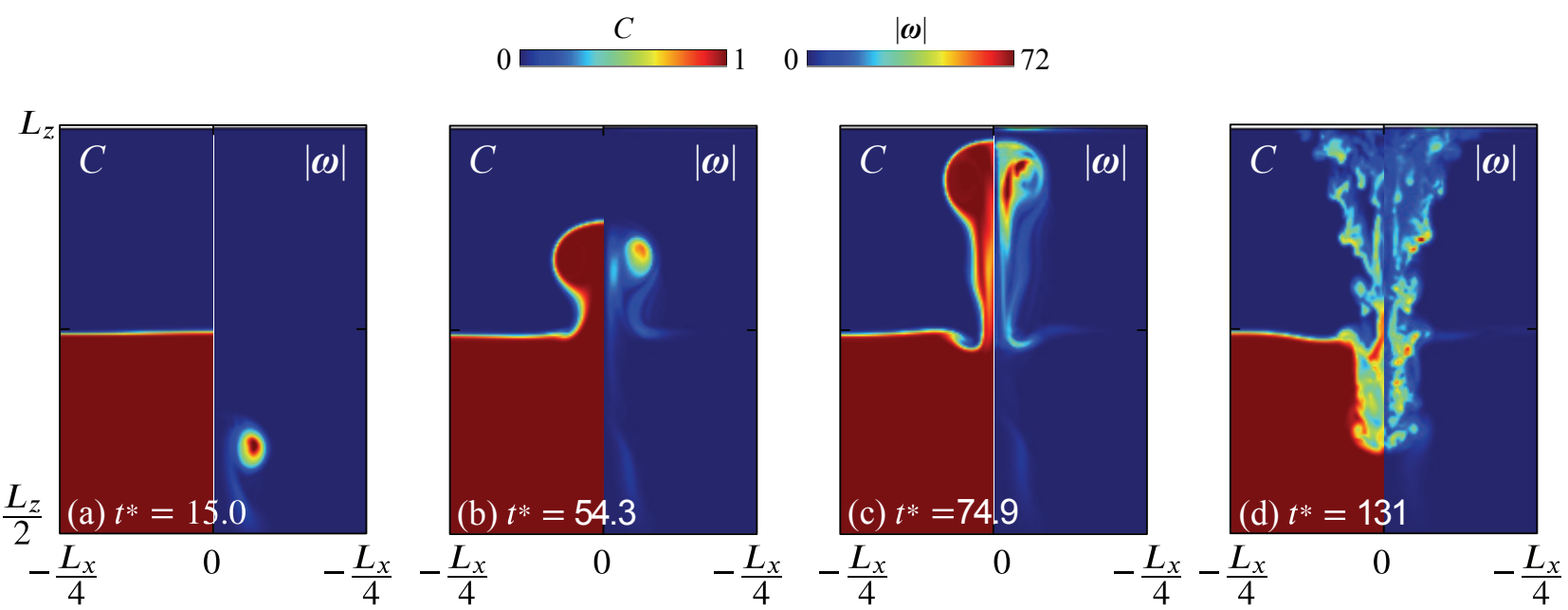

Fig. 7 Time variation of vorticity magnitude and concentration in the central vertical cross-section at $R e=8,000$. 
points with $\operatorname{Re}=4,000$ or 6,000 . In the figures, the vorticity magnitude in the same region $\left(-L_{x} / 4 \leq x \leq\right.$ $0, L_{z} / 2 \leq z \leq L_{z}$ ) as the concentration is visualized and the images are flipped around the vertical $(z-)$ axis. Thus, the left half shows the concentration and the right half shows the vorticity magnitude from $x=0$. During the initial stage, the density interface above the vortex ring rises locally as the vortex ring approaches. The density interface is then entrained into the vortex ring, and the upper fluid is entrained into the lower fluid. High vorticity is generated along the entrained interface. After the vortex ring begins to sink, the vortex ring collapses and the upper fluid is entrained into the lower layer. At this Reynolds number, mixing is mainly caused by this entrainment. At $\operatorname{Re}=6,000$, the vortex ring reaches a higher position than that at $\operatorname{Re}=4,000$, which transports some of the lower fluid into the upper layer. The upper fluid is entrained toward the center of the tank. In this case, mixing is generated by the spreading in the upper layer and the entrainment of the upper fluid into the lower layer after the collapse of the vortex ring. Fig. 7 shows the concentration of the lower fluid and the vorticity magnitude in the central vertical $(x-z)$ cross-section passing through the tank. At $\mathrm{Re}=8,000$, the vortex ring collides with the upper wall of the tank. The vortex ring collides with the wall and spreads widely in the upper layer. This spreading weakens the entrainment of the upper fluid into the lower layer. In this case, mixing is mainly generated in the upper layer by the spreading of the lower fluid transported in the vortex ring.

The vortex ring in the $x-z$ plain was also traced using the center of vorticity obtained from following equation:

$$
\left(x_{c}, z_{c}\right)=\left(\frac{\int_{A}\left|\omega_{y}\right| x z^{2} d A}{\int_{A}\left|\omega_{y}\right| z^{2} d A}, \frac{\int_{A}\left|\omega_{y}\right| x^{2} z d A}{\int_{A}\left|\omega_{y}\right| x^{2} d A}\right)
$$

When the entire region of the $x-z$ plain $\left(-L_{x} / 2 \leq x \leq L_{x} / 2,0 \leq z \leq L_{z}\right)$ is chosen as the integration area, the center of vorticity corresponding to the center of the vortex ring can be calculated while the vortex ring keeps its shape. When half regions of the $x-z$ plain $\left(-L_{x} / 2 \leq x \leq 0,0 \leq z \leq L_{z}\right.$ or $\left.0 \leq x \leq L_{x} / 2,0 \leq z \leq L_{z}\right)$ are chosen as the integration area, the center of the ring core is obtained. The former is referred to as the "vorticity center" and the latter is referred to as the "core center", respectively. Although the vortex ring collapses as it collides with the density interface, the names "vorticity center" and "core center" are used for convenience in describing the calculations in what follows.

Fig. 8 shows the trajectories of the vorticity center and core centers at each Reynolds number. The vorticity center is plotted with a solid line and the core centers are plotted with dashed lines. At $\mathrm{Re}=4,000$, the vortex ring rises up the density interface locally and then convects into the upper region as shown in Fig. 5. But the trajectory of the vorticity center, shown in Fig. 8a, is suppressed just below the height of the density interface $\left(z=3 L_{z} / 4\right)$, because of the vorticity generated around the density interface that is entrained into the vortex ring. The core centers then move outward toward the side walls of the tank, causing fluctuation of the density interface. With $\mathrm{Re}=6,000$, shown in Fig. 8b, the vorticity center and the core centers rise above the initial height of the density interface, but the maximum height is lower than the top of the vortex ring shown in Fig. 6. The vortex ring is also confirmed to be vertically stretched in the upper layer, thus the vorticity center remains lower than the top of the ring. The core centers then move outward above the density interface. In contrast to the results at $\mathrm{Re}=4,000$, the vortex ring collapses in the upper layer so that the lower fluid transported by the ring spreads within the upper layer. The trajectories at $\mathrm{Re}=8,000$ in Fig. 8c reach a higher position. Like the simulations at $\operatorname{Re}=6,000$, the core centers move outward above the density interface, but they reach a higher position than those at $\mathrm{Re}=6,000$ because the vorticity is more distributed vertically. 


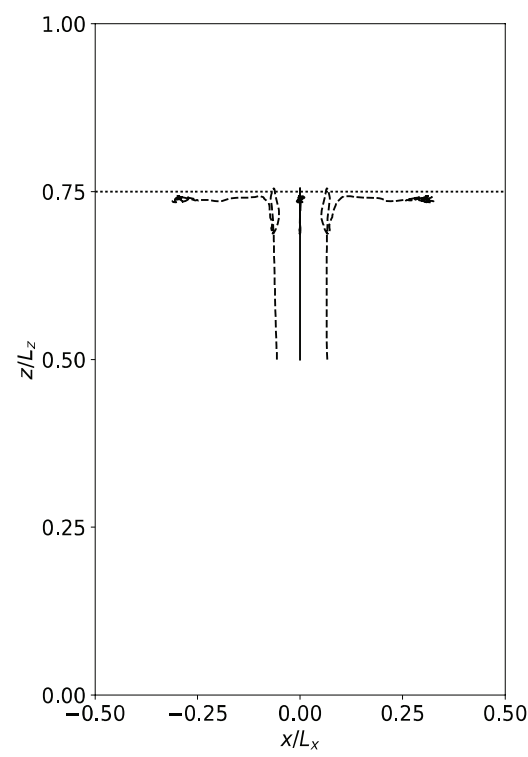

(a) $\operatorname{Re}=4,000$

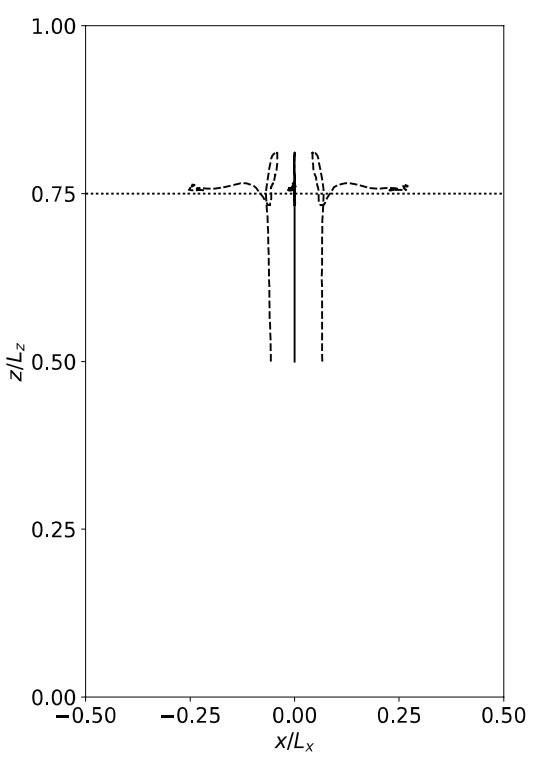

(b) $\operatorname{Re}=6,000$

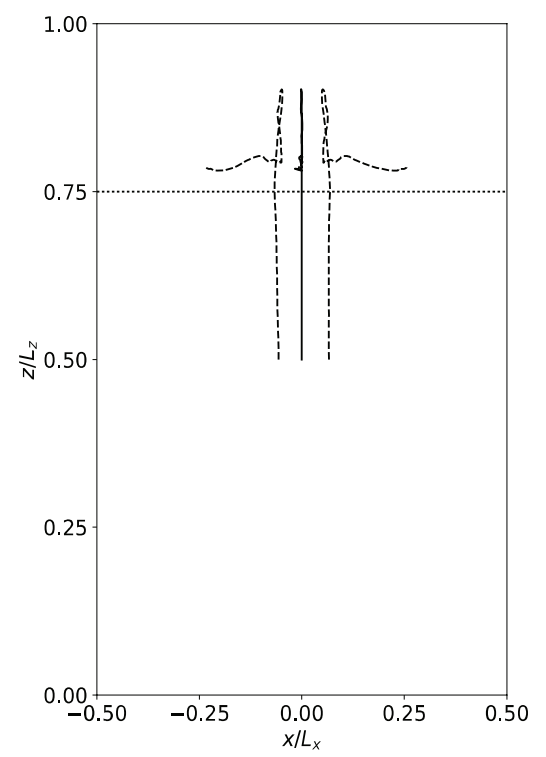

(c) $\operatorname{Re}=8,000$

Fig. 8 Trajectories of the center of the vortex ring and ring's cores.

Fig. 9 shows the change in the elevation of the center for the vortex ring. Initially, the center of the vortex ring moves toward the density interface at a nearly constant speed for every Reynolds number. Fig. 9 also shows the vortex ring displacements with its own self-induced velocity using a formula by Saffman

$$
U=\frac{\Gamma}{4 \pi R}\left[\ln \left(\frac{8 R}{a}\right)-0.558\right]
$$

and using a formula with arbitrary constant $\sigma$ by Tinaikar et al. [24]

$$
U=\frac{\Gamma_{0}}{4 \pi R_{0}\left(1+\frac{\sigma v_{K} t}{a_{0}^{2}}\right)^{3 / 2}}\left[\ln \left(\frac{8 R_{0}}{a_{0}}\right)-0.558\right]
$$

where subscript 0 represents values at the initial state. In the Saffman's formula, the self-induced velocity from the initial values is calculated. The displacement of the vortex ring in the present simulation initially agrees with that from Saffman's formula, but the displacement from Saffman's formula and the simulation gradually depart from each other. The simulation results show good agreement with the result from the formula by Tinaikar et al. with $\sigma=6$.

At $\operatorname{Re}=4,000$, the vorticity center rises slightly higher than the initial height of the density interface.
The vorticity center then briefly descends with the collapse of the vortex ring and the resultant entrainment of the upper fluid into the lower layer. After that, the vorticity center fluctuates just below the density interface. The time variation at $\operatorname{Re}=6,000$ is like the results at $\mathrm{Re}=4,000$, but the vorticity center reaches a higher position and fluctuates just above the density interface. At $\mathrm{Re}=8,000$, the vorticity center reaches $90 \%$ the height of the tank. The vorticity center then descends and fluctuates at a position higher than the density interface. This means that relatively strong vorticity appears in the upper layer due to the spreading of the lower fluid transported by the vortex ring into the upper layer. The vorticity center fluctuates slightly with a long period.

Fig. 10 shows the time variation of the core centers in the horizontal $(x-)$ direction. Just after the collision of the vortex ring with the density interface, the core centers approach each other because the vortex ring is stretched vertically by the entrainment of the density interface. The core centers then part and move toward the side walls of the tank. This motion is derived by the fluctuations of the density interface and their outward propagation. 


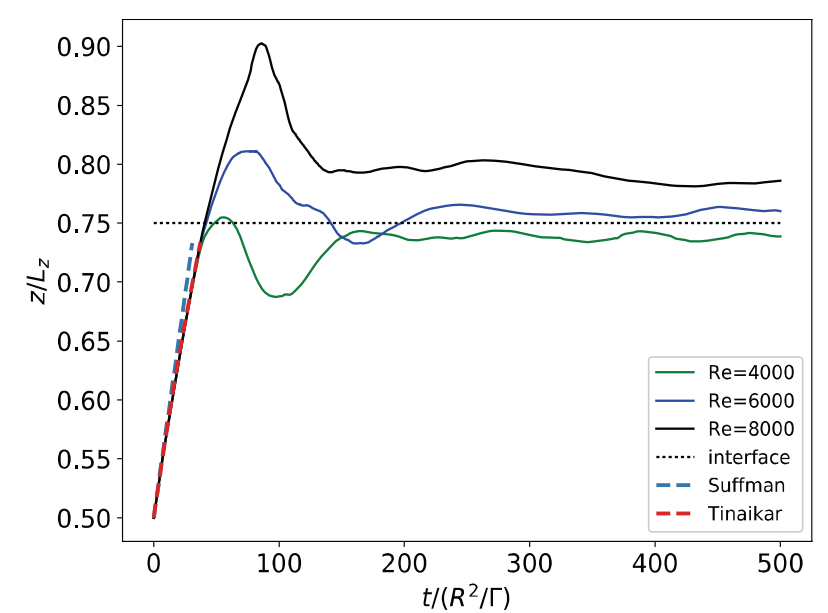

Fig. 9 Time history of the center of the vortex ring.

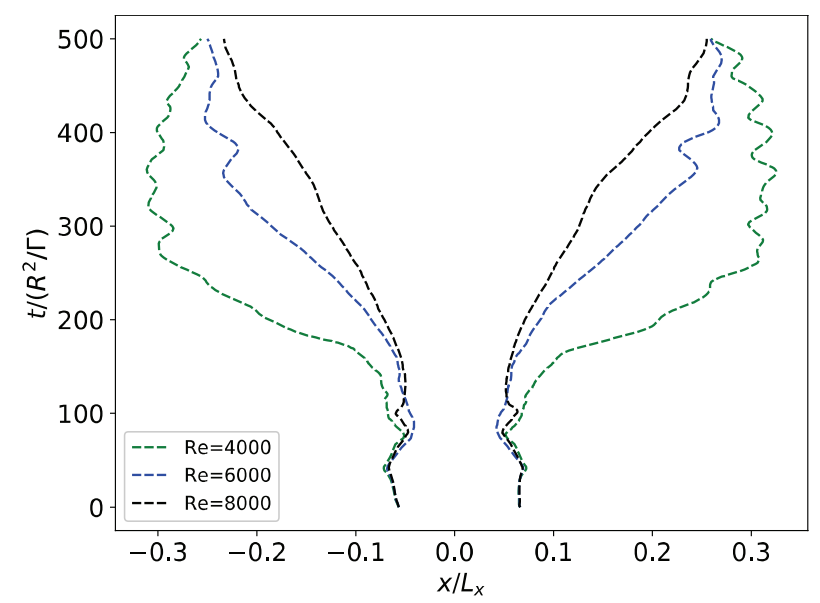

Fig. 10 Time history of the centers of vortex ring's cores.

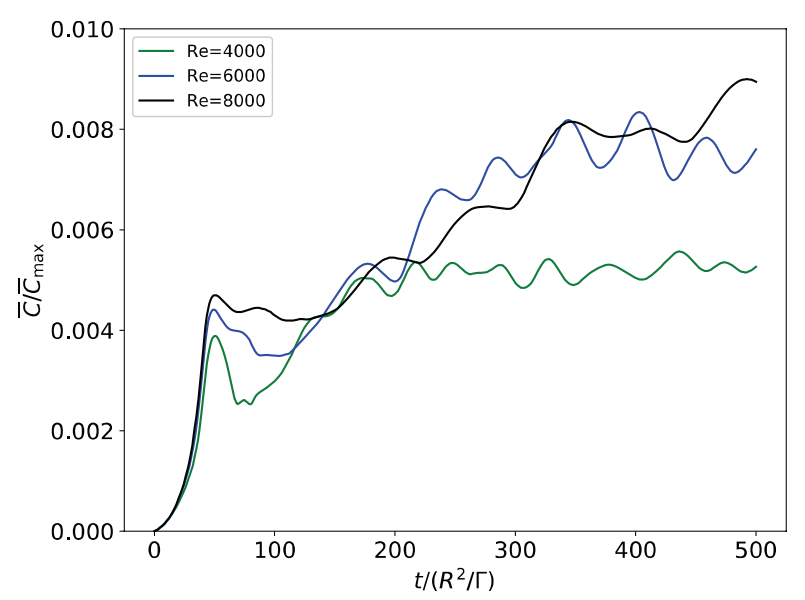

Fig. 11 Time variation of mixing index $\bar{C}$.

\subsection{Progress of Mixing}

The upper and lower fluids are mixed due to the vortex motion in each case. To estimate the progress of mixing in the tank, the mixing index is calculated by the following equation:

$$
\bar{C}=\sum_{k=1}^{N_{z}}\left|\left\langle C_{0}(k)\right\rangle_{x, y}-\langle C(k)\rangle_{x, y}\right|
$$

where $C_{0}$ is the initial concentration of the lower fluid, $k$ is the point coordinate in the $z$-direction, $N_{z}$ is the number of the grid points in $z$-direction. $\langle C(k)\rangle_{x, y}$ is the average value of the concentration on the horizontal $(x-y)$ section at the height of $k \Delta z$. It is defined as:

$$
\langle C(k)\rangle_{x, y}=\frac{1}{N_{x} N_{y}} \sum_{j=1}^{N_{y}} \sum_{i=1}^{N_{x}} C_{i, j, k}
$$

$i, j$ are the point coordinates in the $x-, y$-directions, and $N_{x}, N_{y}$ are the numbers of grid points in the $x-, y-$ directions, respectively.

Fig. 11 shows the time variation of the mixing index $\bar{C}$ at $\operatorname{Re}=4000,6000$, and 8000 , where $\bar{C}$ is normalized by the maximum value of $\bar{C}, \bar{C}_{\text {max }} \cdot \bar{C}$ increases quadratically as the vortex ring rises toward the density interface. $\bar{C}$ decreases after reaching a maximum values because of the sinking motion of the vortex ring. Then $\bar{C}$ increases again because of the entrainment of the upper fluid caused by the collapse of the vortex ring. In the final stage, $\bar{C}$ fluctuates with the heaving motion of the density interface. When the Reynolds number is 8,000 , the decrement of $\bar{C}$ is smaller than that at other Reynolds numbers because the vortex ring collides with the upper wall of the tank and therefore does not sink back down to the density interface.

The absolute value of the concentration gradient vector, $|G|$, is calculated to estimate instantaneous mixing at a point in the fluid field.

$$
|G|=\sqrt{\left(\frac{\partial C}{\partial x}\right)^{2}+\left(\frac{\partial C}{\partial z}\right)^{2}}
$$

This equation is well known as the Prewitt filter, and is used in the field of image processing to extract the edges of objects in pictures. $|G|$ becomes large at positions where the concentration gradient is large. 
Figs. 12-14 show the spatial distributions of $|G|$, where $|G|$ is normalized by the initial maximum value of $|G|,|G|_{\max }$. Until the vortex ring collapses, the value of $|G|$ takes approximately 0 or 1 and the distribution of $|G|$ corresponds to the density interface. After the vortex ring collapses, an intermediate value of $|G|$ appears. The appearance of the intermediate value of $|G|$ implies that mixing is generated by the vortex motion.

Time variations of the spatially integrated value of $|G|, \bar{G}$, are shown in Fig. 15. Since this value becomes large when the concentration gradient is widely distributed, high values represent an inhomogeneous concentration distribution, indicating successful mixing. Differently from the mixing index $\bar{C}$ which increases just after the simulation is started, $\bar{G}$ is constant at first. $\bar{G}$ increases after the vortex ring collides with the density interface and then decreases over time. At $\operatorname{Re}=4,000, \bar{G}$ is almost 1 at $t^{*}>300$. This means that mixing does not occur during this period. At $\mathrm{Re}=6,000$ and $8,000, \bar{G}$ increases to almost the same value, but the gradient of the decrease at $\operatorname{Re}=6,000$ is larger than that at $\operatorname{Re}=$ $8,000 . \bar{G}$ at $\operatorname{Re}=6,000$ is almost 1 at $t^{*}=500 . \bar{G}$ at $\operatorname{Re}=8,000$ is larger than 1 even at $t^{*}=500$, indicating that mixing still occurs.

$$
0 \longdiv { | \boldsymbol { G } | / | \boldsymbol { G } | _ { \operatorname { m a x } } } 1
$$
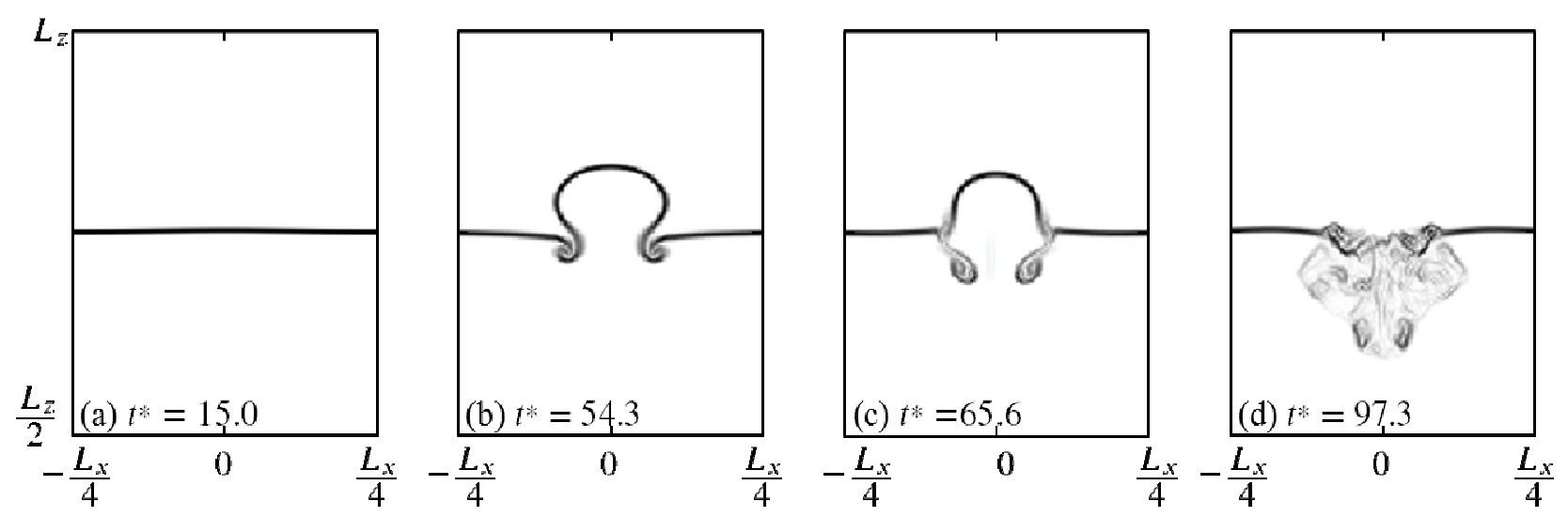

Fig. 12 Distributions of absolute values of the density gradient at $\operatorname{Re}=4,000$.

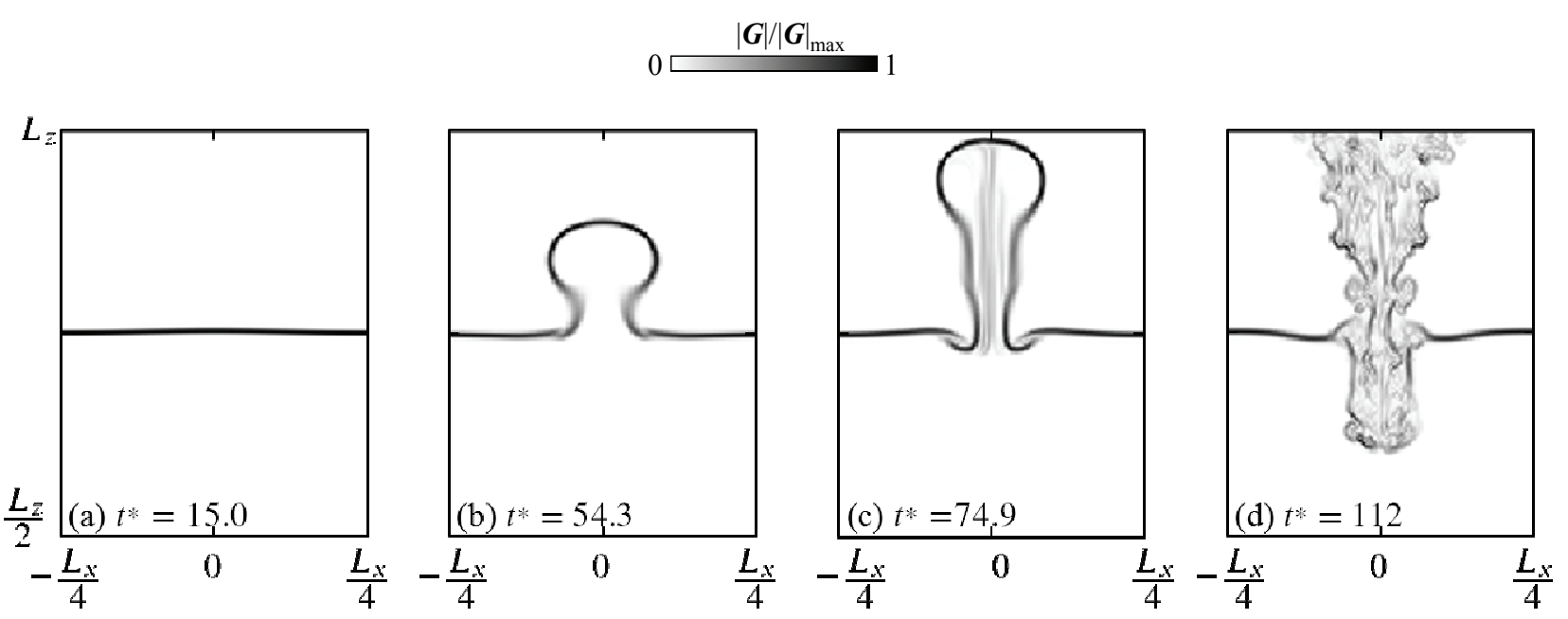

Fig. 13 Distributions of absolute values of the density gradient at $\operatorname{Re}=\mathbf{6 , 0 0 0}$. 

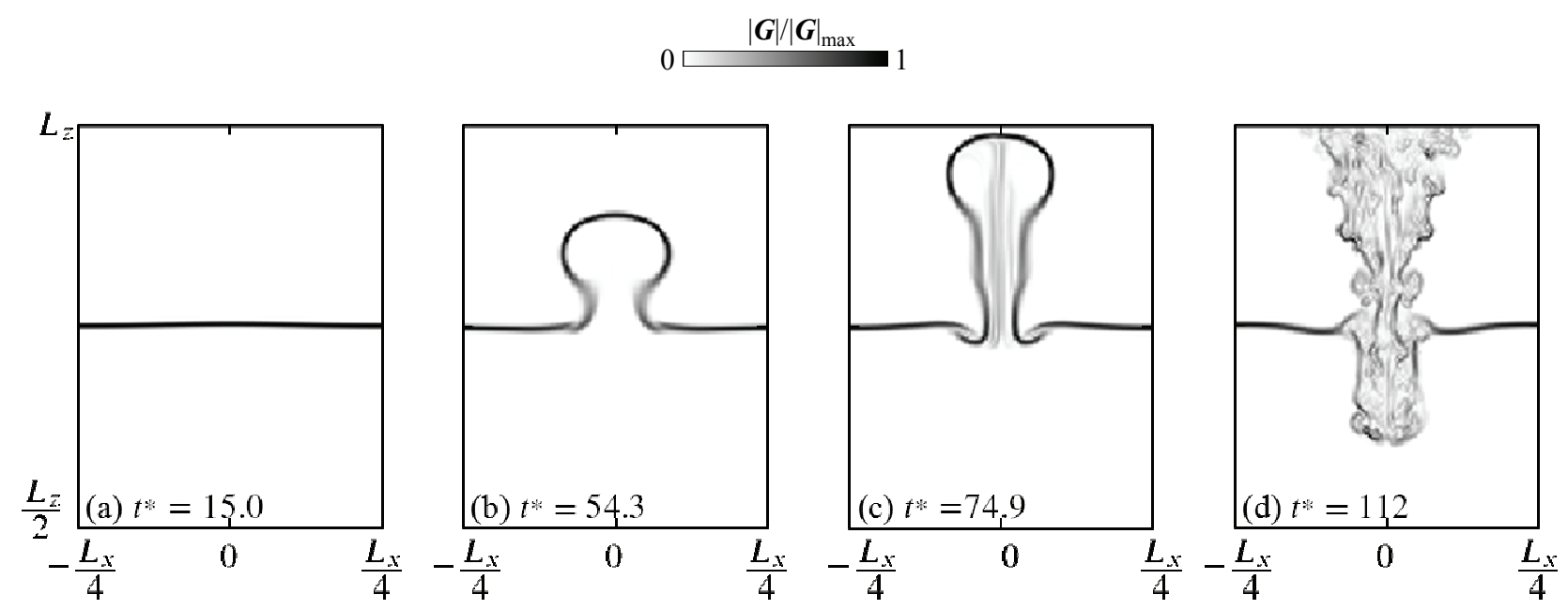

Fig. 14 Distributions of absolute values of the density gradient at $\operatorname{Re}=\mathbf{8 , 0 0 0}$.

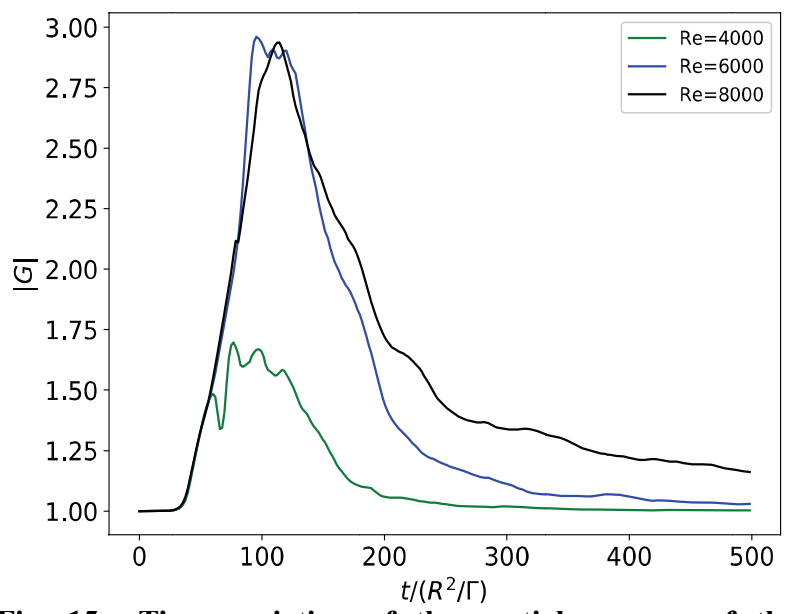

Fig. 15 Time variation of the spatial average of the absolute values of density gradients.

\section{Conclusions}

The behavior of a vortex ring launched vertically upward into a two-layer density-stratified fluid in a rectangular tank was simulated to investigate the mixing phenomena. The upper and lower fluids were an aqueous glycerol solution and an aqueous solution of potassium dihydrogen phosphate $\left(\mathrm{KH}_{2} \mathrm{PO}_{4}\right)$, respectively. The phenomena of vortex rings with Reynolds numbers of 4000, 6000, and 8000 were simulated. The vortex-in-cell method was used to calculate the dynamics of the upper and lower fluids, and the CIP method was used to trace the concentration of the lower fluid.

The results clarify the vortex-ring mixing process, and suggest that an optimal Reynolds number of the vortex ring for the efficient mixing can be found. The type of mixing process due to a vortex ring launched from the bottom of the tank depends on the Reynolds number of the vortex ring. In the case that Reynolds number is relatively low, mixing is generated by the entrainment of the upper fluid into lower layer following the collapse of the vortex ring in the upper layer. With higher Reynolds-number vortex rings, the mixing is mainly generated in the upper layer by the spreading of the lower fluid transported by the vortex rings.

\section{Acknowledgment}

The authors would like to express their gratitude to Hiroyuki Tsunoda, an Associate Professor at Yamanashi University, for providing measurement data of the kinetic viscosity and density of aqueous glycerol and aqueous $\mathrm{KH}_{2} \mathrm{PO}_{4}$ solutions. This study was partially supported by the grant-in-aid for the Project of Creation of Life Innovation Materials for Interdisciplinary and International Researcher Development of the Ministry of Education, Culture, Sports, Science and Technology, Japan.

\section{References}

[1] Baines, W. D., Turner, J. S., and Campbell, I. H. 1990. "Turbulent Fountains in an Open Chamber." J. Fluid 
Mech. 212: 557-92.

[2] Bloomfield, L. J., and Kerr R. C. 1998. "Turbulent Fountains in a Stratified Fluid." J. Fluid Mech. 358: 335-56.

[3] Lin, Y. J. P., and Linden, P. F. 2005. "The Entrainment due to a Turbulent Fountain at a Density Interface." $J$. Fluid Mech. 542: 25-52.

[4] Ansong, J. K., Kyba, P. J., and Sutherland, B. R. 2008. "Fountains Impinging upon a Density Interface." J. Fluid Mech. 595: 115-39.

[5] Shakouchi, S., Fukue, S., and Uchiyama, T. 2015. "Investigation of the Behavior of a Jet Issued into Two-layer Density-stratified Fluid.” J. Flow Control, Meas. Vis. 3: 1-9.

[6] Shakouchi, S., Shimada, S., and Uchiyama, T. 2015. "Numerical Simulation of Jet Flow Issued into Density-stratified Fluid by Vortex in Cell Method." Adv. Appl. Fluid Mech. 17: 115-34.

[7] Degawa, T., Fukue, S., Uchiyama, T., Ishikawa, A., and Motoyama, K. 2017. "Behavior of a Jet Issued Diagonally Upward into Two-layer Density-stratified Fluid in a Cylindrical Tank." J. Flow Control, Meas. Vis. 5: 51-64.

[8] Degawa, T., Uchiyama, T., Aozasa, I., Ishikawa, A., and Motoyama, K. 2018. "Numerical Simulation of Jet Issuing Diagonally Upward into Density-stratified Fluid in Cylindrical Tank." J. Power Energy Eng. 6: 38-52.

[9] Domon, K., Ishihara, O., and Watanabe, S. 2000. "Mass Transport by a Vortex Ring." J. Phys. Soc., Japan 69: 120-3.

[10] Uchiyama, T., Yano, C., and Degawa, T. 2017. "Generation and Transport of Solid Particle Clusters Using a Vortex Ring Launched into Water." Int. J. Chem. Eng. Appl. 8: 253-60.

[11] Bernal, L. P., Maksimovic, P., Toinsi, F., and Tryggvason, G. 1994. "An Experimental and Numerical Investigation of Drop Formation by Vortical Flows in Microgravity." AIAA Paper 94-0244.

[12] Bernal, L. P., and Maksimovic, P. 1996. "The Propagation of a Vortex Ring through Multiple Liquid Interface in
Microgravity.” AIAA Paper 96-0593.

[13] Linden, P. F. 1973. "The Interaction of a Vortex Ring with a Sharp Density Interface: A Model for Turbulent Entrainment." J. Fluid Mech. 60: 467-80.

[14] Olsthoorn, J., and Dalziel, S. B. 2015. "Vortex-ring-induced Stratified Mixing." J. Fluid Mech. 781: 113-26.

[15] Tsunoda, H., Hosaka, M., and Amano, S. 2011. "Visualization of a Round Vortex Ring Interacting with a Density Interface." Trans. Vis. Soc. Japan 31: 21-6. (In Japanese)

[16] Dahm, W. J. A., Scheil, C. M., and Tryggvason, G. 1989. "Dynamics of Vortex Interaction with a Density Interface." J. Fluid Mech. 205: 1-43.

[17] Song, M., Bernal, L. P., and Tryggvason, G. 1992. "Head-on Collision of a Large Vortex Ring with a Free Surface." Phys. Fluids A 4: 1457-66.

[18] Marcus, D. L., and Bell, J. B. 1994. "Numerical Simulation of a Viscous Vortex Ring Interaction with a Density Interface." Phys Fluids 6: 1505-14.

[19] Mihailescu, M., and Gabor, R. A. 2011. "Diffusion coefficient of Potassium Dihydrogen Phosphate Using Holographic Interferometry.” Rom. J. Phys. 56: 399-410.

[20] Cottet, G.-H., and Koumoutsakos, P. D. 2000. Vortex Methods. New York: Cambridge University Press.

[21] Takewaki, H., Nishiguchi, N., and Yabe, T. 1985. "The Cubic Interpolation Pseudo-particle (CIP) Method for Solving Hyperbolic-type Equations.” J. Comput. Phys. 61: 261-8.

[22] Kim, D., Song, O. Y., and Ko, H. S. 2008. "A Semi-Lagrangian CIP Fluid Solver without Dimensional Splitting." Comput. Graphics Forum 27: 467-75.

[23] Uchiyama, T., Yoshii, Y., and Hamada, H. 2015. "Direct Numerical Simulation of a Turbulent Channel Flow by an Improved Vortex in Cell Method." Int. J. Numer. Methods Heat Fluid Flow 24: 103-23.

[24] Tinaikar, A., Advaith, S., and Basu, S. 2018. "Understanding Evolution of Vortex Rings in Viscous Fluids." J. Fluid Mech. 836: 873-909. 